\title{
Brain-Derived Neurotrophic Factor Signaling in the HVC Is Required for Testosterone-Induced Song of Female Canaries
}

\author{
Tessa E. Hartog, ${ }^{1}$ Falk Dittrich, ${ }^{2}$ Anton W. Pieneman, ${ }^{1}$ René F. Jansen, ${ }^{1}$ Carolina Frankl-Vilches, ${ }^{2}$ Volkmar Lessmann, ${ }^{3}$ \\ Christina Lilliehook, ${ }^{4}$ Steven A. Goldman, ${ }^{4}$ and Manfred Gahr ${ }^{2}$ \\ ${ }^{1}$ Institute for Neuroscience, VU University Amsterdam, 1081 HV Amsterdam, The Netherlands, ${ }^{2}$ Max Planck Institute for Ornithology, 82319 Seewiesen, \\ Germany, ${ }^{3}$ Institute for Physiology, Otto von Guericke University, D-39120 Magdeburg, Germany, and ${ }^{4}$ University of Rochester Medical Center, Rochester, \\ New York 14642
}

Testosterone-induced singing in songbirds is thought to involve testosterone-dependent morphological changes that include angiogenesis and neuronal recruitment into the HVC, a central part of the song control circuit. Previous work showed that testosterone induces the production of vascular endothelial growth factor (VEGF) and its receptor (VEGFR2 tyrosine kinase), which in turn leads to an upregulation of brain-derived neurotrophic factor (BDNF) production in HVC endothelial cells. Here we report for the first time that systemic inhibition of the VEGFR2 tyrosine kinase is sufficient to block testosterone-induced song in adult female canaries, despite sustained androgen exposure and the persistence of the effects of testosterone on HVC morphology. Expression of exogenous BDNF in HVC, induced locally by in situ transfection, reversed the VEGFR2 inhibition-mediated blockade of song development, thereby restoring the behavioral phenotype associated with androgen-induced song. The VEGFR2-inhibited, BDNF-treated females developed elaborate malelike song that included large syllable repertoires and high syllable repetition rates, features known to attract females. Importantly, although functionally competent new neurons were recruited to $\mathrm{HVC}$ after testosterone treatment, the time course of neuronal addition appeared to follow BDNF-induced song development. These findings indicate that testosterone-associated VEGFR2 activity is required for androgen-induced song in adult songbirds and that the behavioral effects of VEGFR2 inhibition can be rescued by BDNF within the adult HVC.

\section{Introduction}

In oscine songbirds, testosterone $(\mathrm{T})$ and its estrogenic metabolites are involved in both the developmental and seasonal acquisition of song (Gurney and Konishi, 1980; DeVoogd and Nottebohm, 1981). In a well studied model system, male canaries (Serinus canaria) sing seasonally in a testosterone-dependent manner to attract females (Nottebohm et al., 1987; Voigt and Leitner, 2008). Female canaries do not sing at all during the breeding season but may produce a relatively primitive song, known as subsong outside of the breeding season. When treated with testosterone, female canaries can develop highly structured songs that resemble those of reproductively active males (Heid et al., 1985; Fusani et al., 2003). The first vocalizations of these testosterone-treated females are subsongs that are highly variable. Over a period of several weeks, however, the spectral-temporal structure of the songs becomes more stereotyped, developing sequences of identically repeated syllables, called tours. Such testosterone-induced song is associated with gross anatomic changes to the neural circuit controlling song (Nottebohm,

Received June 2, 2009; revised 0ct. 21, 2009; accepted 0ct. 27, 2009.

This work was supported by The Netherlands Organisation for Scientific Research, Division Earth and Life Sciences Grant 813.07 .004 (T.E.H.) and National Institute of Neurological Disorders and Stroke Grant R3729813 (S.A.G.). We thankDr. V. Kodelja for help with primer designs and Dr. M. Konishiand A. ter Maat for comments on this manuscript.

Correspondence should be addressed to Manfred Gahr, Max Planck Institute for Ornithology, D-82319 Seewiesen, Germany. E-mail: gahr@orn.mpg.de.

D01:10.1523/JNEUROSCI.2564-09.2009

Copyright $(\odot 2009$ Society for Neuroscience $\quad 0270-6474 / 09 / 2915511-09 \$ 15.00 / 0$
1980), an interconnected set of testosterone-responsive central nuclei known as the song control system (Nottebohm et al., 1976). Among the principal relay nuclei of the vocal control system is HVC, a pallial center of the dorsomediocaudal forebrain. In adult songbirds, HVC is involved in the generation of higherorder song patterns such as syllable sequences ( $\mathrm{Yu}$ and Margoliash, 1996; Hahnloser et al., 2002). Remarkably, HVC of domesticated canaries exhibits seasonal plasticity associated with song acquisition and extinction (Nottebohm et al., 1986; Gahr, 1990) and is sexually dimorphic, exhibiting a larger volume and more complex neuropil in singing males than in nonsinging females (Nottebohm, 1980). However, the causal relationship of testosterone-dependent neural plasticity to song development has remained unclear (Gahr, 1990, 2004).

Testosterone-driven structural plasticity of the adult song control system is associated with both a vascular expansion within HVC and the associated recruitment by HVC of new neurons from the subependymal zone (Goldman and Nottebohm, 1983; Louissaint et al., 2002). The onset of angiogenesis before neuronal recruitment in the HVC of testosterone-treated canaries suggests that the HVC vasculature might act as a source of both chemoattractive and neurotrophic factors that serve to direct neuronal addition to the adult HVC (Louissaint et al., 2002). Accordingly, studies of the androgen-treated adult $\mathrm{HVC}$ revealed that testosterone initially induces the production of both vascular endothelial growth factor (VEGF) and its receptor, the VEGFR2/ flk1 tyrosine kinase, which leads to rapid endothelial cell division 
and consequently to an expansion of the HVC microvasculature. The endothelial cells within the newly expanded HVC vasculature produce brain-derived neurotrophic factor (BDNF), which in turn supports the differentiation therein of newly generated neurons (Louissaint et al., 2002). Importantly, blockade of endothelial cell division by an inhibitor of the VEGFR2 tyrosine kinase suppressed not only angiogenesis but also testosterone-associated neuronal addition (Louissaint et al., 2002).

In the present study, we first asked whether the systemic inhibition of VEGFR2 tyrosine kinase, and the consequent suppression of angiogenesis and neuronal addition to HVC, is sufficient to block both the testosterone-dependent development of the song system and the attendant acquisition of testosteroneinduced song by female canaries (see Fig. $1 A, B$ ). We then asked whether this inhibition of song could be reversed by local BDNF overexpression, acting as a downstream effector of neuronal addition to HVC. We found that the development of song in response to testosterone treatment could indeed be blocked by pharmacological inhibition of HVC angiogenesis and neurogenesis, although testosterone exerts its inductive action on HVC morphology, and that song could be rescued by the expression of BDNF in the HVC of VEGFR2-inhibited, testosterone-treated birds.

\section{Materials and Methods}

\section{Housing}

Female canaries were moved from aviaries into recording cages $(56 \times$ $28 \times 38.5 \mathrm{~cm})$ placed inside sound-proofed boxes and maintained on spring-like photoperiod (14/10 h light/dark cycle). Water and food were available ad libidum. Each box was equipped with a microphone that was connected to a preamplifier and a National Instruments PCI 6071E 12bit 16-channel analog-to-digital converter. Sound-triggered data acquisition was used to automatically record all acoustic events (calls and songs). Files were saved as time-stamped uncompressed digital audio files to a hard disk with a sampling rate of $44,052 \mathrm{~Hz}$. All audio files were manually checked and selected for the presence of songs using soundanalysis software.

\section{Experimental procedures}

Our experimental design is summarized in Figure 1. Untreated females were first recorded for 2 weeks to ensure that they were not singing spontaneously. Afterward, between experimental days 1-7 (Fig. 1), birds were injected intraperitoneally twice daily with $50 \mu \mathrm{g}$ of 5-bromo-2'deoxyuridine (BrdU) per gram of body weight (Sigma) in $0.9 \%$ saline. On day 8, HVC was tranfected bilaterally with a BDNF plasmid expression vector (100 nl/hemisphere) under isoflurane anesthesia. Additional females were given BDNF plasmid injections bilaterally into the robust nucleus of the arcopallium (RA) or into the nidopallium adjacent to the HVC. To control for the surgery and the plasmid injection, other females underwent either a sham operation or were injected with an empty plasmid not containing the BDNF sequence into the HVC. After surgery, all birds were implanted with SILASTIC tubes (Dow Corning) containing either testosterone propionate (Sigma) or nothing (empty SILASTICs). Between days 8 and 34, females were injected intramuscularly with either VEGFR2-I (inhibitor of vascular endothelial growth factor receptor tyrosine kinase) (4-[(4'-chloro-2'-fluoro)phenylamino]-6,7-dimethoxyquinazoline [Calbiochem]; $2.5 \mu \mathrm{g}$ of VEGFR2-I per gram of body weight) dissolved in vehicle (DMSO/PBS, 2:1) or either the DMSO/PBS vehicle or PBS only. Because vehicle and PBS females did not differ in their measurements, we collectively refer to them as PBS-treated throughout this paper. At day 26, all SILASTIC implants were renewed. On day 44 , the birds were killed by an overdose of isoflurane and perfused transcardially with $50 \mathrm{ml}$ each of $0.9 \%$ saline and $4 \%$ formaldehyde solution. The brains were removed, postfixed in $4 \%$ formaldehyde for 1 week, and then cryoprotected in 10\% sucrose for $5 \mathrm{~d}$ and $20 \%$ sucrose for $3 \mathrm{~d}$. The right hemisphere was cut on a freezing microtome into $40 \mu \mathrm{m}$ sagittal sections, and the left hemisphere was stored at $-80^{\circ} \mathrm{C}$.

An additional set of females ("BDNF only") was injected with BrdU and given BDNF plasmid as above but was not treated with either testosterone or VEGFR2-I. Another set of females was treated as above, except for the date when the animals were killed (four "T+VEGFR2-I+BDNF" females and four "T+VEGFR2-I" females; the latter received an empty plasmid). Each of the latter was randomly assigned to one of the former, and each female was maintained single in a sound box. One day after start of singing of the T+VEGFR2-I+BDNF females, these females and their nonsinging matched control females were killed.

\section{Expression plasmid and in vivo transfection}

Expression plasmid. Plasmid vector was designed to express the rat BDNF under cytomegalovirus (CMV) promoter control. Two stop codons were introduced between the coding sequences of the BDNF and enhanced green fluorescent protein (eGFP) in an expression plasmid (p-eGFP-N1; Clontech) coding for the corresponding C-terminal eGFP-tagged fusion protein (pCMV-BDNF-eGFP) (Haubensak et al., 1998). Thus, expression of the rat BDNF could be detected by PCR (supplemental results, available at www.jneurosci.org as supplemental material) and in situ hybridization using primers and probes, respectively, directed against the eGFP sequence of the BDNF-stop-stop-eGFP-mRNA, which excludes the detection of endogenous BDNF (Fig. 1C).

Plasmid DNAs were purified from Escherichia coli K12 (JM109) cells and used for in vivo transfection at a concentration of $3 \mu \mathrm{g} / \mu \mathrm{l}$ when incubated with ExGen 500 in vivo transfection reagent (MBI Fermentas). For the preparation of the transfection solution, instructions of the manufacturer were followed. Briefly, $21 \mu \mathrm{g}$ of plasmid DNA were diluted in $50 \mu \mathrm{l}$ (final volume) of sterile $20 \mathrm{~mm}$ HEPES buffer at $\mathrm{pH} 7.4$ with $5 \%$ glucose. Then, $3.6 \mu \mathrm{l}$ of ExGen 500 in vivo transfection reagent was added, and the resulting solution was immediately mixed thoroughly and centrifuged at 13,000 rpm for $1 \mathrm{~min}$ at room temperature (RT). After incubating for $10 \mathrm{~min}$ at RT, $50 \mathrm{nl}$ of the transfection solution was pressure injected bilaterally into HVC of anesthetized animals.

Area-specific distribution of the BDNF plasmid. For the detection of BDNF-eGFP-mRNA in HVC by in situ hybridization, parasagittal cryostat sections $(20 \mu \mathrm{m})$ were hybridized with sense and antisense probes for eGFP following standard protocols (Fig. 1C) (supplemental Fig. S1, available at www.jneurosci.org as supplemental material). To produce the probes, an eGFP fragment of 379 bp was amplified from p-eGFPN1 with a forward (CACATGAAGCAGCACGATTT) and a reverse (TGCTCAGGTAGTGG TTGTCG) primer. The PCR product was cloned into the pGEMT cloning vector (Promega). Orientation and sequence of the insert in this construct was verified by DNA sequencing. The vector pGEMT-eGFP was linearized with the restriction enzymes NotI and NcoI and used as template for the synthesis of radioactive sense and antisense RNA probes, respectively. For both probes, the radioactivity applied to each slide was $8 \times 10^{6} \mathrm{cpm}$. For signal detection, sections were exposed together for 3 weeks to an autoradiographic film (Kodak Biomax MR) from which microphotographs were taken. For all time points, the sense probe resulted in a much weaker signal compared with the antisense probe (data not shown).

Western blot analysis. The effect of BDNF-eGFP transfection on the level of BDNF protein in HVC was investigated in adult male zebra finches by Western blot analysis (Fig. $1 D$ ). Among birds killed 0-26 d after in vivo transfection, parasagittal brain sections of $300 \mu \mathrm{m}$ were prepared at $4^{\circ} \mathrm{C}$ with a vibratome, and HVC tissue was dissected under direct visualization through an inverted microscope. For each time point, transfected HVC tissue of two brain sections was collected, homogenized in SDS sample buffer, separated on a $10-20 \%$ gradient gel by SDS-PAGE, and blotted onto a Hybond-ECL nitrocellulose membrane (GE Healthcare). BDNF was detected as its precursor protein (proBDNF) with a polyclonal rabbit antibody (PP1000P; Acris), using peroxidase-labeled anti-rabbit IgG (Vector Laboratories) as the second antibody. proBDNF exhibits a major doublet band at $\sim 30 \mathrm{kDa}$, which respectively represent the glycosylated and nonglycosylated forms of proBDNF (Pang et al., 2004). Membranes were incubated with the ECL Western Blotting Analysis System (GE Healthcare) and exposed to Kodak BioMax XAR films. 

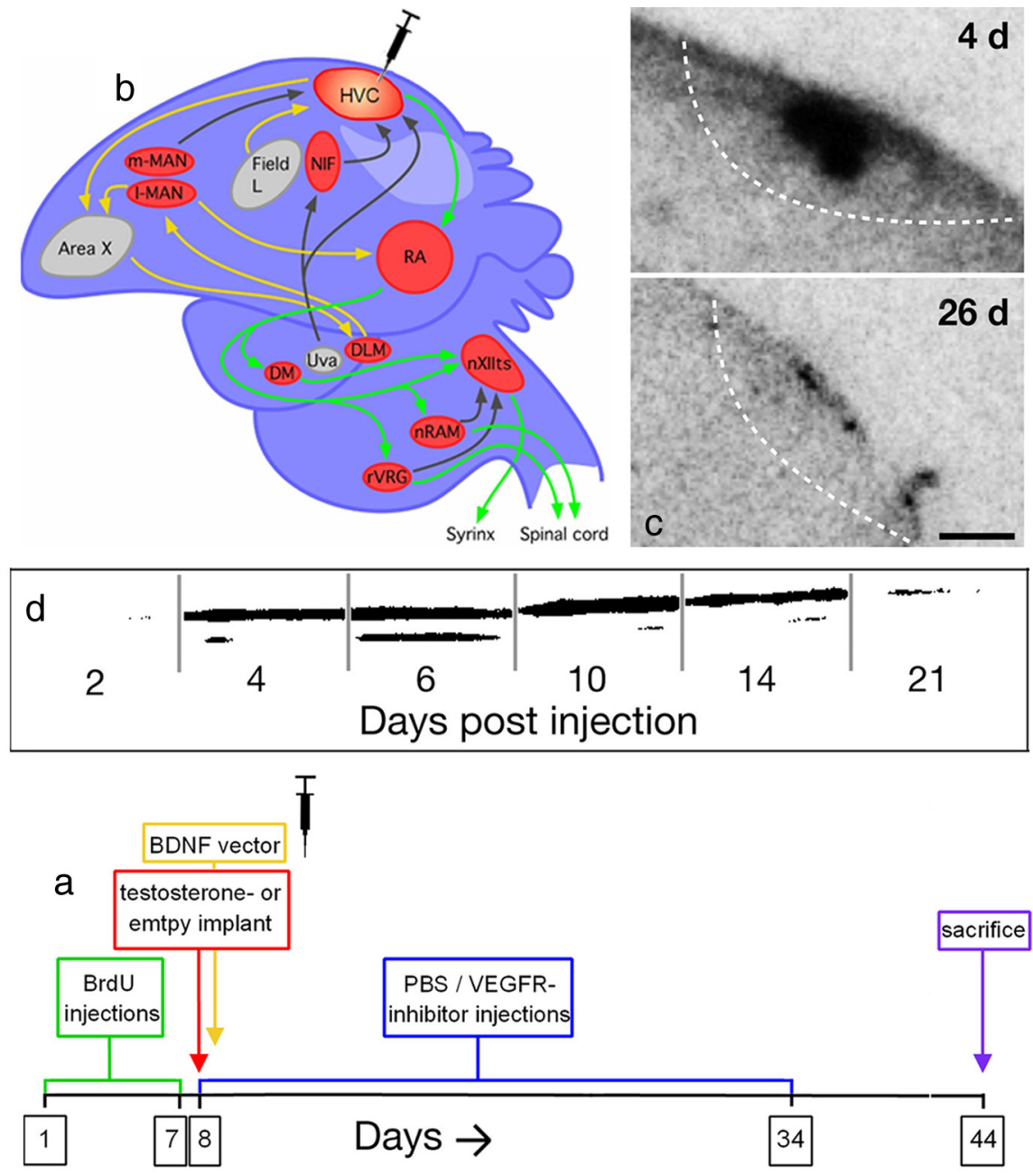

Figure 1. Experimental design $(\boldsymbol{a}, \boldsymbol{b})$ and BDNF expression of HVC $(\boldsymbol{c}, \boldsymbol{d}) \cdot \boldsymbol{a}$, The experimental design (for details, see Materials and Methods, Experimental procedures). $\boldsymbol{b}$, BDNF-expressing plasmid was transfected into nucleus HVC of the song control system, shown in a schematic sagittal view. The motor pathway (green arrows) is involved in song production and ultimately innervates the bird's sound-producing organ, the syrinx. The anterior forebrain pathway (yellow arrows) is an internal feedback system thought to be involved in song learning. Nuclei depicted in red express androgen receptors. HVC expresses both androgen and estrogen receptors (for additional details and abbreviations, see Bolhuis and Gahr, 2006). c, Local distribution of the BDNF plasmid in the HVC. Microphotographs of autoradiograms of sections that were obtained from males killed 4 and $26 \mathrm{~d}$ after in vivo transfection, respectively, and hybridized with a radioactive antisense probe for BDNF- eGFP. The black labeled areas are those that contain the BDNF- eGFP-mRNA. The dashed lines indicate the ventral border of HVC. $\boldsymbol{d}$, Time course of expression of BDNF precursor protein (proBDNF) in the HVC as determined by Western blot analysis. The intensity of a major doublet band detectable at $29 \mathrm{kDa}$ that is characteristic for proBDNF was relatively increased to controls at $4-14 \mathrm{~d}$ after in vivo transfection. Peak expression was between 6 and $10 \mathrm{~d}$, in agreement with the rBDNF-GFP-mRNA expression kinetics shown in supplemental Figure S1 (available at www. jneurosci.org as supplemental material).

\section{Immunolabeling and morphometry}

HVC and RA volumes were determined from Nissl-stained sections based on their cytoarchitecture; although Nissl-stained borders of the female HVC can be somewhat ambiguous (Gahr, 1990), we did not stain for additional Nissl-independent markers because the adjacent HVC sections were needed for immunostaining. In brief, every fourth section was mounted onto slides, allowed to dry, and then Nissl stained $(0.1 \%$ thionin; Sigma). The slides were viewed under a microscope (Leica Leitztype DMRBE FLV/photo TV) equipped with a camera (Leica Leitz HB050L1; Visitron Systems) and digitized to measure area size using SPOT software (Visitron Systems). The HVC and RA volume of each brain was calculated by these area measurements, the section thickness, and the interval between the sections.

Neuron staining. To quantify neuron numbers, we performed neuronal-specific nuclear protein $(\mathrm{NeuN})$ immunostaining on free-floating sections as described previously (Mullen et al., 1992). Briefly, sections were washed, treated with a blocking solution (0.1 м PBS, $1 \%$ BSA, and $0.3 \%$ Triton $\mathrm{X}-100$ ) for $30 \mathrm{~min}$, and then incubated with anti-NeuN (MAB377; Millipore Bioscience Research Reagents) diluted 1:250 in the blocking solution at $4^{\circ} \mathrm{C}$ for $48 \mathrm{~h}$. Thereafter, sections were washed in PBS and treated with the secondary antibody (biotinylated goat anti-mouse IgG; Dako) diluted 1:200 in the blocking solution for $2 \mathrm{~h}$. After washing in PBS, sections were incubated in ExtrAvidinperoxidase conjugate (Sigma) diluted 1:500 in blocking solution for $2 \mathrm{~h}$. The sections were washed again, treated with DAB (Sigma)/ $\mathrm{H}_{2} \mathrm{O}_{2}$, washed, mounted on slides, and dried before coverslipping with Entellan (Merck). To estimate the total number of neurons in HVC and RA of each animal, the numbers of neurons were counted in three counting volumes (each $560.7 \mu \mathrm{m}^{3}$ ) within three sections of either HVC or RA. The total neuron number of HVC and RA was derived from the average of these measurements and the volume of HVC and RA, respectively.

$B r d U$ and ZENK staining. Concurrent BrdU/ ZENK/4',6' -diamidino-2-phenylindole (DAPI) immunostaining was done on free-floating sections that included HVC. The sections were permeabilized in $0.2 \%$ Triton X-100 in PBS for $30 \mathrm{~min}$, incubated in $0.1 \mathrm{~N} \mathrm{HCl}$ for $20 \mathrm{~min}$ at $37^{\circ} \mathrm{C}$, neutralized with Trizma base $(12 \mathrm{~g} / \mathrm{L}$ $\mathrm{H}_{2} \mathrm{O}, \mathrm{pH}$ 8.5; Sigma) twice for $5 \mathrm{~min}$, washed, and then incubated in 20\% normal goat serum (NGS) in PBS for 30 min. Next, sections were incubated in a 1:100 dilution of mouse antiBrdU IgG (347580; BD Biosciences) in PBS at $4^{\circ} \mathrm{C}$ for $60 \mathrm{~h}$, washed in PBS, and incubated in a 1:400 dilution of biotinylated goat anti-mouse IgG (E0433; Dako) in PBS for $2 \mathrm{~h}$. After washing, sections were incubated in a $5 \mu \mathrm{g} / \mathrm{ml}$ PBS dilution of streptavidin-FITC for $1 \mathrm{~h}$ (S3762; Sigma). The sections were washed, treated with 5\% NGS in PBS for $20 \mathrm{~min}$, and incubated in 1:500 rabbit anti-egr1 (synonym of ZENK) IgG (sc-189; Santa Cruz Biotechnology) in PBS at $4^{\circ} \mathrm{C}$ for $20 \mathrm{~h}$. After washing, sections were blocked with 5\% NGS in PBS for 20 min, incubated in a 1:400 dilution of Alexa Fluor 594 goat anti-rabbit IgG in PBS for $1 \mathrm{~h}$ (A11012; Invitrogen), washed again, and immersed in a 1 $\mu \mathrm{g} / \mathrm{ml}$ dilution of DAPI in PBS for $25 \mathrm{~min}$ (Merck). Finally, sections were washed, mounted onto slides, dried, and coverslipped with Vectashield. The sections were examined using a Leica AF6000 microscope system. All neurons that had incorporated BrdU and all ZENK/egr1-immunoreactive BrdU-positive neurons were counted within HVC. HVC can be visualized in the DAPI staining as a result of its lower neuron densities compared with the surrounding tissue.

\section{Sound analysis}

We analyzed all sounds that had been recorded (both calls and songs), using a custom computer program (written by R.F.J.). Files containing song or song-like sounds were studied in detail with respect to the number of syllables and repetition rate (syllables per second). Syllable repertoire and repetition rates were analyzed from at least 50 songs of each bird. Different syllables were detected by inspection of the sonograms considering the fundamental frequency and its harmonics and the frequency modulations, i.e., the "morphology" of the syllable. For each 
tour, a custom software program (written by R.F.J.) calculated the length of syllables, the length of the pauses between syllables, and, from these data, the repetition rate of identical syllables.

\section{Statistical analysis}

All statistical tests were performed using Kyplot version 4.0.1d and JMP IN 5.1. MannWhitney $U$ test was used for comparisons of the behavioral data. One-way ANOVA with Tukey's post hoc $t$ tests for multiple comparisons, using $\alpha=0.05$, was used for the analysis of neuroanatomical data. When two-tailed $t$ tests are used, we have indicated this accordingly and have followed these analyses with a Shapiro-Wilk normality test. Error bars represent SDs, unless stated otherwise.

\section{Results}

Testosterone-induced song is blocked by concurrent inhibition of VEGFR2, an effect reversed by BDNF

We first investigated the effects of blocking angiogenesis on the development of song in T-treated adult female canaries, using an inhibitor of VEGFR2 signaling (Fig. 2). Nonsinging female canaries were divided into several treatment groups (see Materials and Methods). Over the course of the experiment, $\mathrm{T}$ triggered singing in 16 of 19 (84\%) females (T+PBS females). In contrast, only 3 of 15 (20\%) birds implanted with $\mathrm{T}$ but also injected with VEGFR2-I sang (T+VEGFR2-I females). Of the three females of this T+VEGFR2-I group that did develop song, two had the smallest song repertoire of all birds (see below). Treatment of the T-implanted females with vehicle (solvent of VEGFR2-I) did not inhibit singing (7 of 7 sang). In contrast, none of the control females (null females) implanted with empty implants (0 of 17) sang, regardless of whether they were additionally treated with PBS $(n=7)$, vehicle $(n=4)$, or VEGFR2-I $(n=6)$. Together, these results show that VEGF signaling is required for testosterone-induced song.

It was noted previously that testosterone, acting through VEGF as a paracrine mediator, triggers the expression of BDNF by the newly generated HVC microvascular bed (Louissaint et al., 2002). On the basis of this study, we next asked whether BDNF expression is required for testosterone-induced, VEGF-mediated song acquisition. To this end, we sought to determine whether BDNF overexpression is sufficient to sustain testosterone-induced song, despite concurrent VEGFR2 inhibition (Fig. 1C,D). A total of 15 T-implanted birds were transfected intraparenchymally to express BDNF in HVC and were then treated with either PBS or VEGFR2-I. All 15 birds developed song, regardless of whether they had been injected with VEGFR2-I ( $n=8$; T+VEGFR2$\mathrm{I}+\mathrm{BDNF}$ females) or not ( $n=7 ; \mathrm{T}+\mathrm{PBS}+\mathrm{BDNF}$ females) (Fig. $2)$. Furthermore, females $(n=3)$ that were treated with T+VEGFR2-I and an empty plasmid did not sing. Thus, expression of exogenous BDNF in the adult female HVC overcomes the inhibitory effect of systemic VEGFR2-I on testosterone-induced song development. Females that obtained BDNF plasmid transfection into HVC $(n=5)$ but no testosterone implant did not sing at the times when females of all other experimental groups started singing, as reported previously (Rasika et al., 1999).

Interestingly, when analysis was restricted to singing females in each group, we noted that the onset of plastic song occurred in all groups at $\sim 10 \mathrm{~d}$ of testosterone treatment ( $p=0.16$ ) (supplemental Fig. S2, available at www.jneurosci.org as supplemental material). In addition, we noted that BDNF expression served to reduce the latency to stable song in all $\mathrm{T}+\mathrm{BDNF}$ females $(p=$ $0.03)$, regardless of their additional treatment. Importantly, the song facilitating effect of BDNF appeared specific to HVC. Overexpression of BDNF in RA of females that were treated with testosterone and the VEGFR2 inhibitor did not induce song development $(n=3)$. Furthermore, injections of the BDNF plasmid into the nidopallium adjacent to HVC in T+VEGFR2-I females $(n=4)$ yielded no evident effect on song behavior.

\section{VEGFR2 inhibition impedes and BDNF recovers syllable repertoires and syllable repetition rates}

Canary songs are composed of syllables that may be repetitively uttered in series known as tours (Güttinger, 1985). Testosterone affects the length of tours, as well as the syllable complexity used in the overall song repertoire, and their repetition rates (Heid et al., 1985; Nottebohm et al., 1987; Leitner et al., 2001; Fusani et al., 2003). Female canaries respond to songs that are composed of large syllable repertoires with increased nest-building activity 

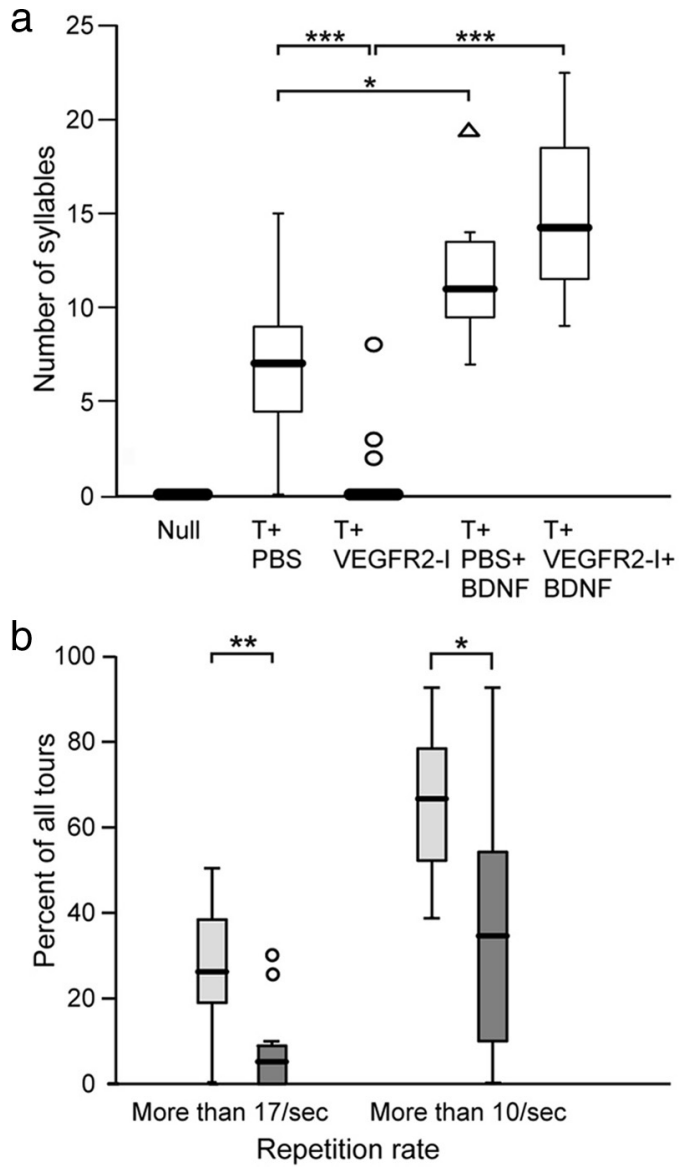

Figure 3. BDNF facilitates the production of large song syllable repertoires $(\boldsymbol{a})$ and high syllable repetition rates $(\boldsymbol{b}) \cdot \boldsymbol{a}$, Note that BDNF treatment (T+VEGFR2-I+ BDNF) overcomes the inhibitory effect of VEGFR2-I (T+VEGFR2-I) on the development of syllable repertoires after testosterone treatment. Females not receiving testosterone (null) do not sing and differed from all other groups ( $p \leq 0.0001$ ). The length of each box shows the range of the central $50 \%$ of the syllable repertoire, with the borders of the box at the first and third quartiles, and the median value represented by a thick line. The whiskers show the range of values that fall within the inner fences. Values between the inner and outer fences are plotted with triangles, and values outside the outer fence are plotted with circles. ${ }^{*} p \leq 0.05 ;{ }^{* *} p \leq 0.001 ;{ }^{* * *} p \leq 0.0001$, by Mann-Whitney $U$ test. $\ln \boldsymbol{b}$, we measured the percentage of tours that are produced with repetition rates above $10 \mathrm{~Hz}$ and above $17 \mathrm{~Hz}$; the latter tours have the potential to be sexually salient (Vallet and Kreutzer, 1995). Testosterone-treated females that obtained BDNF (light boxes) are compared with those not treated with BDNF (dark boxes). The boxes represent the same characteristics as described in $\boldsymbol{a}$. The values between the inner and outer fences are plotted with circles. ${ }^{*} p \leq 0.05 ;{ }^{* *} p \leq 0.001$, by $t$ test.

(Kroodsma, 1976). Furthermore, females respond to songs that contain tours with high syllable repetition rates ( $>17$ times/s) with increased courtship solicitation display, indicating the sexual salience of these song features (Vallet and Kreutzer, 1995).

As a first level of analysis, we counted the number of different syllables produced by each bird (Fig. $3 a$ ). The repertoires of the $\mathrm{T}+\mathrm{PBS}$ and T+VEGFR2-I group, including the nonsingers, differed significantly from one another $(p=0.0001)$. The median number of syllables sung by the T+PBS group was 7 , with first and third quartile values of 4 and 9 , respectively. In contrast, the median number of syllables sung by the T+VEGFR2-I females was 0 , as were the first and third quartiles. Considering only the singing females of both groups, the $\mathrm{T}+\mathrm{PBS}$ females sang, on average, $8.2 \pm 3.1$ different syllables, whereas the T+VEGFR2-I females produced $4.3 \pm 3.2$ syllables. Two of these latter three birds produced the smallest repertoires of all singing females.
In contrast to the suppression of song complexity noted in response to VEGFR2 inhibition in females treated only with testosterone, VEGFR2 inhibition did not diminish syllable numbers in matched birds that were also given an intra-HVC BDNF expression plasmid: $\mathrm{T}+\mathrm{PBS}+\mathrm{BDNF}$ females sang $11.1 \pm$ 4.0 syllables, and T+VEGFR2-I+BDNF birds sang $15.0 \pm 4.5$ different syllables (Fig. $3 a$ ). Whereas application of the BDNFexpressing vector increased syllable repertoire compared with T+VEGFR2-I females ( $p=0.0001)$, injection of the empty plasmid into HVC $(n=3)$ had no such effect in T+VEGFR2-I females $(p=0.9)$. Although the average repertoire size of $\mathrm{T}+\mathrm{PBS}$ females was smaller than that of $\mathrm{T}+\mathrm{PBS}+\mathrm{BDNF}$ females $(p=$ $0.0148)$, this difference seemed in part attributable to the nonsinging females in the T + PBS group. Removal of these nonsinging females resulted in an average syllable repertoire of $8.2 \pm 3.1$, only marginally less than that of the $\mathrm{T}+\mathrm{PBS}+\mathrm{BDNF}$ females $(11.1 \pm 4.0)(p=0.06)$. Thus, BDNF transfection into HVC primarily restored syllable variety in testosterone-treated birds in whom syllable variety had been otherwise suppressed by VEGFR2-I treatment.

We next studied the detailed structure and use of the syllable repertoire, particularly in regards to features associated with sexual attraction, such as rapid syllable repetition rates. We then quantified these endpoint features by calculating the fraction of tours in the songs that were produced below or above a fixed rapid rate, which we defined as $17 \mathrm{~Hz}$ (Fig. 3b). Because the songs of T+PBS+BDNF and T+VEGFR2-I+BDNF females did not differ ( $t$ test, $p=0.4625$ ) we combined these into a single group ( $\mathrm{T}+\mathrm{BDNF}$ females) and compared their syllable repetition rates with the $\mathrm{T}+\mathrm{PBS}$ females. In the $\mathrm{T}+\mathrm{BDNF}$ females, these fast tours comprised $25.9 \pm 14.9 \%$ of the song repertoire, whereas only $7.7 \pm 9.8 \%$ of the songs of T + PBS females included such rapid tours ( $t$ test, $p=0.001$ ). We noted a similar result when we measured the percentage of tours faster than $10 \mathrm{~Hz}(66.7 \pm$ $16.4 \%$ fast tours in $\mathrm{T}+\mathrm{BDNF}$ females vs $36 \pm 28 \%$ in $\mathrm{T}+\mathrm{PBS}$ birds; $t$ test, $p=0.0017$ ). The higher proportion of repetition rates above $10 \mathrm{~Hz}$ of $\mathrm{T}+\mathrm{BDNF}$ females was similar to songs of reproductively active males (T. E. Hartog and M. Gahr, unpublished observations). Thus, BDNF strongly increased the use of rapidly repeated syllables in the songs. Together, these data show that BDNF expression in HVC is important not only for the development of testosterone-dependent song and syllable repertoires but also for the specific production of sexually salient songs.

\section{Testosterone increases HVC neuronal numbers}

Morphometric analysis of T and/or VEGFR2-I treated brains revealed that $\mathrm{T}$ treatment was associated with a $184 \%$ increase in HVC volume, relative to that of untreated null females $(p<$ 0.0001) (Fig. 4a). Concurrent treatment with VEGFR2-I did not influence the effect of T treatment on HVC volume. Furthermore, additional treatment with BDNF had no effect on HVC volume, beyond that already accomplished by testosterone administration $\left(0.241 \pm 0.046 \mathrm{~mm}^{3}\right.$ for $\mathrm{T}+\mathrm{PBS}$ females and $0.202 \pm 0.038 \mathrm{~mm}^{3}$ for $\mathrm{T}+\mathrm{PBS}+\mathrm{BDNF}$ females) (Fig. $4 a$ ). Similarly, BDNF had no significant effect on HVC volume in testosterone-treated birds treated with VEGFR2-I; HVC volumes measured $0.211 \pm 0.036 \mathrm{~mm}^{3}$ in T+VEGFR2-I females versus $0.218 \pm 0.049 \mathrm{~mm}^{3}$ in T+VEGFR2-I+BDNF females. Control null females exhibited HVC volumes of $0.133 \pm 0.027 \mathrm{~mm}^{3}$ (Fig. $4 a)$. We also measured the volumes of the RA, which exhibits strong angiogenesis but no neurogenesis in response to $\mathrm{T}$, and found analogous treatment effects as those noted in HVC (sup- 
a

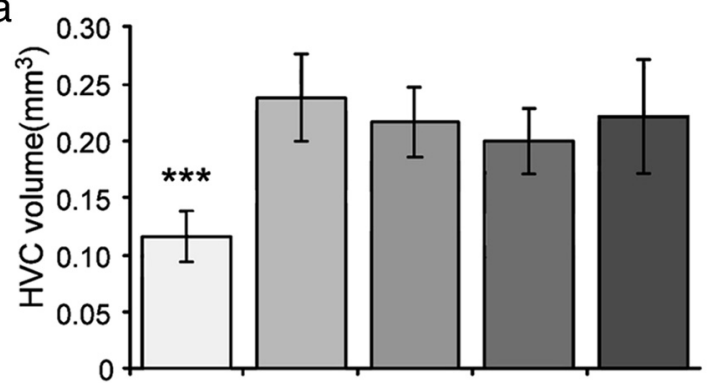

b

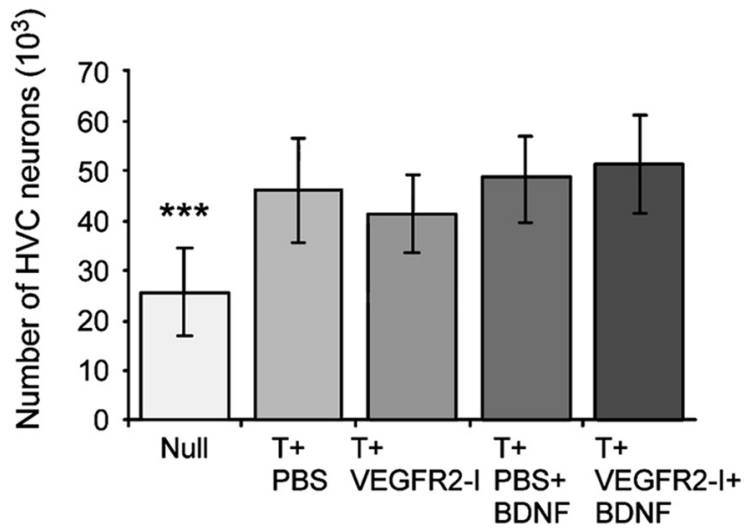

Figure 4. Effect of testosterone on HVC volume (a) and HVC neuron numbers ( $\boldsymbol{b})$. $\boldsymbol{a}$, Testosterone treatment increases the HVC volume compared with null females (no testosterone). This effect is not impaired by additional treatment with VEGFR2-I (T+VEGFR2-I) and/or BDNF $(T+P B S+B D N F$ and $T+V E G F R 2-I+B D N F) . \boldsymbol{b}$ shows that testosterone increases the total number of HVC neurons compared with null females independent of additional treatments with BDNF and/or VEGFR2-I. ${ }^{* * *} p \leq 0.0001$, by one-way ANOVA.

plemental Results, available at www.jneurosci.org as supplemental material).

Thus, whereas testosterone induces the differentiation of both the adult HVC and RA, VEGFR2 tyrosine kinase inhibition and its attendant suppression of angiogenesis and neuronal recruitment did not influence the gross anatomical change of HVC in response to testosterone. Next, for estimation of neuron numbers, sections were stained with NeuN, which is selectively expressed by neurons (Mullen et al., 1992). We estimated HVC neuron numbers (Fig. $4 b$ ) on the basis of the NeuN-positive neuronal density and the net volume of HVC. We found that testosterone treatment increased the total number of HVC neurons from $25,576 \pm 8808$ in null females to $45,938 \pm 7854$ neurons in $\mathrm{T}+\mathrm{PBS}$ birds $(p<0.0001)$. Treatment of T+PBS females with VEGFR2-I (T+VEGFR2-I females) or with VEGFR2-I and BDNF (T+VEGFR2-I+BDNF females) had no effect on HVC neuron numbers (Fig. $4 b$ ).

\section{VEGFR2 tyrosine kinase inhibition suppressed neuron recruitment}

Testosterone-dependent neuron recruitment into HVC requires BDNF (Rasika et al., 1999; Louissaint et al., 2002). Accordingly, we noted significantly fewer BrdU-labeled neurons (Fig. 5) in $\mathrm{T}+$ VEGFR2-I females than in $\mathrm{T}+\mathrm{PBS}, \mathrm{T}+\mathrm{PBS}+\mathrm{BDNF}$, or $\mathrm{T}+$ VEGFR2-I+BDNF females $(p<0.0001)$ (Fig. $5 b)$. Conversely, there was no significant difference between $\mathrm{T}+\mathrm{PBS}+\mathrm{BDNF}$ and $\mathrm{T}+$ VEGFR2-I+BDNF females in the total number of new HVC neurons, suggesting that the inclusion of BDNF was sufficient to negate the effects of VEGFR2-I on HVC neuronal number (Fig. 5b). a

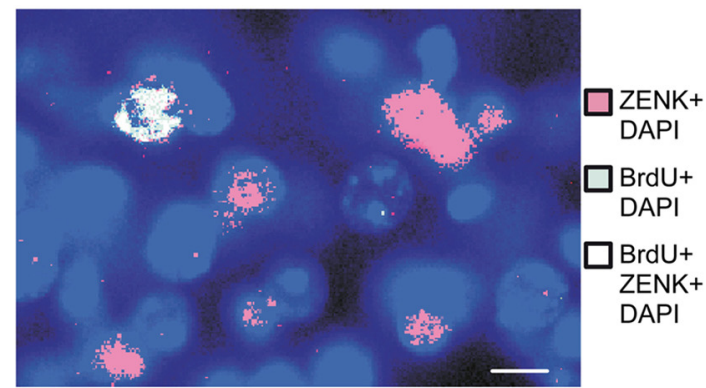

b
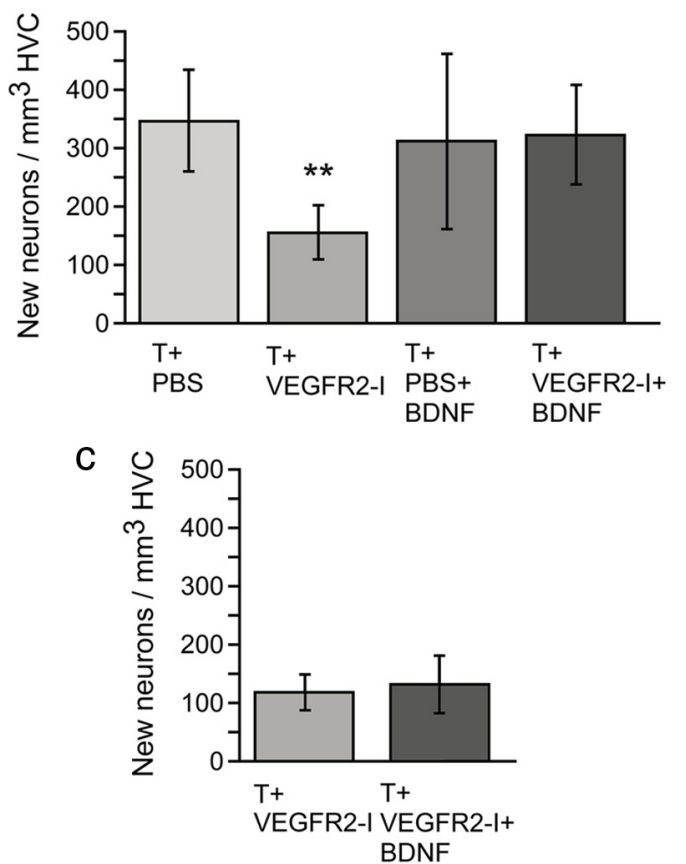

Figure 5. The recruitment of new functional HVC neurons is subsequent to BDNF-dependent singing activity. $\boldsymbol{a}$, In the HVC of adult female canaries, we observed neurons triple labeled for the immediate early gene ZENK (red), the cell birth marker BrdU (green), and the DNA marker DAPI (blue). These neurons are newly born and functional, as indicated by ZENK expression after singing. Scale bar, $10 \mu \mathrm{m} . \boldsymbol{b}$, After $40 \mathrm{~d}$ of testosterone treatment, the number of new functional neurons is smaller in females treated with testosterone and VEGFR2-I (T+VEGFR2-I) than in such females, in which we expressed exogenous BDNF locally in HVC (T+VEGFR2I+BDNF). ${ }^{* *} p \leq 0.0001$, by one-way ANOVA. $c$, The number of new neurons of singing T+VEGFR2-I+BDNF females and of nonsinging T+VEGFR2-I females is similar at the beginning of song development, $9-13 \mathrm{~d}$ after testosterone treatment started.

Because the T+VEGFR2-I females not only recruited fewer new neurons but also failed to sing, we evaluated whether the BDNF-induced recruitment of new neurons was a prerequisite for singing or, rather, followed song development. To this end, we quantified the number of new HVC neurons at the onset of singing in both $\mathrm{T}+\mathrm{VEGFR} 2-\mathrm{I}+\mathrm{BDNF}$ females and in timematched T+VEGFR2-I females that did not sing. T+VEGFR2I+BDNF females began to sing between 9 and $13 \mathrm{~d}$ after testosterone treatment, whereas the T+VEGFR2-I birds failed to sing, similar to the previous results depicted in supplemental Figure S2 (available at www.jneurosci.org as supplemental material). However, these groups did not differ from one another in the number of new neurons found in their HVCs $1 \mathrm{~d}$ after initiating song (Fig. 5 c). Instead, treatment-dependent differences in neuronal number only became apparent at longer posttreatment time points. Thus, the number of new neurons in this T+VEGFR2-I+BDNF group killed $1 \mathrm{~d}$ after manifest- 
ing song (9-13 d after initiating testosterone treatment) was significantly smaller than their counterparts killed $36 \mathrm{~d}$ after initiating testosterone treatment (Tukey's honestly significant difference test at the 0.05 level). In contrast, the number of new HVC neurons in the T+VEGFR2-I females did not differ between the two death time-points (Fig. 5b,c). Because the T+VEGFR2-I group killed at the early time point and three such females of the late time point obtained an empty plasmid, the plasmid transfection per se did not influence the neuron recruitment. These results suggest that the BDNF-dependent mechanisms required for song development preceded the enhanced recruitment of new HVC neurons in testosteronetreated females.

To identify these newly recruited neurons as functional, we combined BrdU labeling with immunodetection of ZENK (egr-1) (Fig. 5a), an activity-dependent immediate early gene that is quickly upregulated in HVC neurons after singing (Jarvis and Nottebohm, 1997; Jin and Clayton, 1997). Of seven BDNFtreated females studied in this manner, three were singing during the $30 \mathrm{~min}$ before they were killed, although four did not do so but could hear the singers. In all seven females, every BrdUpositive HVC neuron scored was observed to coexpress ZENK (Fig. 5), indicating that these new neurons could be activated by singing or hearing song. Because the enhanced recruitment of new HVC neurons in T+VEGFR2-I+BDNF females occurred after the onset of song development, the new neurons may be recruited so as to respond to the birds own auditory stimuli or to enhanced motor-related activity of the HVC.

\section{Discussion}

Testosterone-induced development of the song system appears necessary but not sufficient for singing

We first manipulated both testosterone-induced angiogenesis and neuronal recruitment, while quantitatively assessing testosteroneinduced song development as a measure of behavioral outcome. We found that the typical induction of song in female canaries by testosterone is inhibited by concurrent administration of a VEGFR2 tyrosine kinase inhibitor, a treatment that was shown previously to inhibit transiently the expansion of the HVC microvasculature (Louissaint et al., 2002). Most surprising, the testosterone-induced increase of HVC neuronal numbers and HVC volume differed between controls (null females) and all testosterone-treated groups ( $\mathrm{T}+\mathrm{PBS}, \mathrm{T}+\mathrm{VEGFR} 2-\mathrm{I}$, $\mathrm{T}+\mathrm{PBS}+\mathrm{BDNF}$, and T+VEGFR2-I+BDNF) but was similar among the testosterone-treated groups, regardless of whether they were singing or not. Thus, song development, although dependent on testosterone-induced BDNF, could nonetheless be divorced from both the net volume of HVC and the numbers of its resident neurons. These findings point to testosteronedependent factors that modify the neural architecture of the HVC, which may be suppressed by inhibition of VEGFR2 tyrosine kinase activity. These data, however, must be viewed in the context of two caveats. First, the VEGFR2-I inhibitor of VEGFR2 signaling was given systemically, so that the lack of testosteroneinduced song in these birds might reflect the action of this agent on vocal areas other than HVC or some element of systemic toxicity that may have indirectly impaired HVC function. Second, although the VEGFR2-I used is rather specific for VEGFR2 (Hennequin et al., 1999), this class of tyrosine kinase inhibitors may retain some lesser activity at related receptors, the PDGF and placental growth factor receptors in particular.

To address these concerns, in a second set of experiments, we showed that the effect of testosterone-induced VEGFR2 signaling- associated BDNF on song production was topographically specific to HVC. To this end, we capitalized on the previous observations (1) that testosterone promotes endothelial proliferation in HVC via the VEGF-VEGFR2 pathway and (2) that testosterone induces BDNF production in endothelial cells of $\mathrm{HVC}$ (Louissaint et al., 2002) by expressing BDNF locally in the HVC of otherwise VEGFR2-I-treated females. In agreement with these previous results (Louissaint et al., 2002), angiogenesis was decreased in the HVC of our females during the period of VEGFR2-I treatment (supplemental Results, available at www. jneurosci.org as supplemental material). We found that the expression of exogenous BDNF locally in HVC, but not adjacent to HVC, overcomes the inhibitory effect of VEGFR2-I on testosterone-induced singing behavior of females. Thus, the induction of singing by testosterone requires an intact VEGFR2 signaling pathway in the HVC that, in turn, facilitates BDNF production. Although lesions of the HVC suggest that canaries remain motivated to sing despite HVC injury (Nottebohm et al., 1976), our work strongly indicates that BDNF signaling anchored in HVC is necessary for singing to occur. Thus, we propose that elevated levels of BDNF in HVC are not only a consequence of singing ( $\mathrm{Li}$ et al., 2000) but also the cause of initial song activity. Interestingly, although BDNF is necessary for the development of male-typical song, it is not sufficient to induce singing, because infusing BDNF protein into the HVC of adult female canaries not treated with testosterone failed to induce singing (Rasika et al., 1999). To an extent, the lack of song in females treated only with BDNF might reflect the importance of the testosterone-induced hypertrophy of the syringeal musculature (Bleisch et al., 1984), in providing a physical substrate for vocal execution. More broadly, however, these data suggest that an HVC morphology shaped by the action of testosterone is a prerequisite for BDNF to activate those central mechanisms that lead to singing.

Interestingly, the songs of the T+VEGFR2-I+BDNF females were similar to, or even more structured than, the songs of females treated only with testosterone. This in turn suggests that BDNF produced in HVC may have differentially facilitated the differentiation of mature song features, such as more song segments repeated with high speed, known to be sexually salient (Vallet and Kreutzer, 1995). This notion is supported by the observation that BDNF expression can be modulated by many factors besides sex hormones, which reflect environmental conditions (Dittrich et al., 1999; Louissaint et al., 2002; Scharfman and Maclusky, 2005; Berton et al., 2006). In this regard, female canaries whose ability to convert testosterone to estrogen was blocked lacked BDNF expression in their HVCs and produced songs with limited fast repetition (Fusani et al., 2003). Together with the observation by Louissaint et al. (2002) that estradiol is necessary for the upregulation of VEGFR2 in endothelial cells, these observations suggest that estrogenic activation of the HVC vascular bed may be a critical determinant of song structure and complexity.

\section{Recruitment of new HVC neurons follows song development} The cellular mechanisms that are induced by the BDNF signaling in the HVC, and which in turn lead to singing, are difficult to pinpoint because BDNF plays an important role in so many distinct processes, including synaptic function and plasticity, as well as neuronal recruitment (Rasika et al., 1999; Huang and Reichardt, 2001; Louissaint et al., 2002; Yamada and Nabeshima, 2003). Because BDNF compensated for the inhibitory effects of VEGFR2-I on the recruitment of new functional HVC neurons in 
our females, we discuss in the following the role of neuron recruitment for song development.

The transiently active expression plasmid supports exogenous BDNF production for 2-3 weeks (Fig. 1), which overlaps with the period of highest bioactivity of infused BDNF protein on longterm survival of new HVC neurons (Alvarez-Borda et al., 2004). In untreated canaries, new neurons assume a sedentary phenotype in HVC within 8-15 d after birth but become part of descending premotor pathway in $\sim 1$ month (Kirn et al., 1999). Song development of our females started $\sim 10 \mathrm{~d}$ after and malelike song patterns were achieved $\sim 20 \mathrm{~d}$ after the start of the treatment with testosterone and BDNF. Thus, song development preceded enhanced neuron recruitment into the HVCs of the T+VEGFR2-I+BDNF females. The present data together with previous observations ( $\mathrm{Li}$ et al., 2000; Louissaint et al., 2002) suggest the following scenario of testosterone-dependent neuron recruitment and song development. Testosterone expands the endothelial bed of the HVC via the VEGFR2 pathway. The endothelial cells produce BDNF under the influence of testosterone and its metabolites. Because BDNF overexpression in RA and in the nidopallium outside HVC has no effect on singing, the behavioral activity of BDNF is at least in part unfolding in HVC, although retrograde and anterograde mechanisms in the song control system are possible. This BDNF drives some unknown mechanisms in HVC that facilitates singing. Singing leads to a sustained increase of (neuronal) BDNF, which enhances the recruitment of new HVC neurons, i.e., the motor-related activity of the HVC of BDNF-transfected females itself might be responsible for the neuron recruitment rather than the BDNF. The BDNFdependent recruitment of new neurons in the T+VEGFR2-I females indicates that this action of testosterone is not mediated via VEGFR2 directly, although VEGFR2 signaling might be neurogenic (Jin et al., 2002).

Although the number of new HVC neurons in our animals was relatively small, they are expressing the immediate early gene ZENK, which is otherwise upregulated in HVC neurons of vocalizing songbirds (Jarvis and Nottebohm, 1997). Our behavioral data suggest that these new neurons play a specific role in patterning the HVC activity rather than in song development per se. Indeed, the newly recruited HVC neurons may be involved in consolidation of song motor memories, because the "T+VEGR2-I+BDNF" females appear to crystallize their songs when the new HVC neurons are 3-4 weeks old. Nonetheless, syllable repetition rates were higher in testosterone-treated females that also received BDNF, although the number of new neurons was similar in the T+PBS and T+VEGR2-I+BDNF females. Thus, neuron recruitment in the bird song control system may be important for specific aspects (to be determined) of vocal patterning or communication rather than for song acquisition per se. This observation is reminiscent of recent experimental efforts to define the behavioral significance of neuron recruitment in the adult mammalian hippocampus and olfactory system. These studies have shown that experimentally suppressed neuronal recruitment becomes behaviorally manifest only under very specific testing circumstances, whereas overall behavioral performance such as olfactory perception and spatial navigation are generally normal (Cao et al., 2004; Meshi et al., 2006; Saxe et al., 2006, 2007; Dupret et al., 2008; Farioli-Vecchioli et al., 2008; Imayoshi et al., 2008; Clelland et al., 2009). These results may reflect both the complex sensory tasks of the olfactory bulb and the multifunctionality of the hippocampus, which is involved in tasks as diverse as spatial learning and navigation, behavioral and endocrine stress responses, novelty detection, and contextual fear conditioning (Miller and McEwen, 2006). Similarly, whereas the HVC is involved in a well defined and highly orchestrated behavior, singing, its dependence on the addition of new neurons may be limited to quite discrete aspects of song learning and execution.

\section{References}

Alvarez-Borda B, Haripal B, Nottebohm F (2004) Timing of brain-derived neurotrophic factor exposure affects life expectancy of new neurons. Proc Natl Acad Sci U S A 101:3957-3961.

Berton O, McClung CA, Dileone RJ, Krishnan V, Renthal W, Russo SJ, Graham D, Tsankova NM, Bolanos CA, Rios M, Monteggia LM, Self DW, Nestler EJ (2006) Essential role of BDNF in the mesolimbic dopamine pathway in social defeat stress. Science 311:864-868.

Bleisch W, Luine VN, Nottebohm F (1984) Modification of synapses in androgen-sensitive muscle. I. Hormonal regulation of acetylcholine receptor number in the songbird syrinx. J Neurosci 4:786-792.

Bolhuis JJ, Gahr M (2006) Neural mechanisms of birdsong memory. Nat Rev Neurosci 7:347-357.

Cao L, Jiao X, Zuzga DS, Liu Y, Fong DM, Young D, During MJ (2004) VEGF links hippocampal activity with neurogenesis, learning and memory. Nat Genet 36:827-835.

Clelland CD, Choi M, Romberg C, Clemenson GD Jr, Fragniere A, Tyers P, Jessberger S, Saksida LM, Barker RA, Gage FH, Bussey TJ (2009) A functional role for adult hippocampal neurogenesis in spatial pattern separation. Science 325:210-213.

DeVoogd T, Nottebohm F (1981) Gonadal hormones induce dendritic growth in the adult avian brain. Science 214:202-204.

Dittrich F, Feng Y, Metzdorf R, Gahr M (1999) Estrogen-inducible, sexspecific expression of brain-derived neurotrophic factor mRNA in a forebrain song control nucleus of the juvenile zebra finch. Proc Natl Acad Sci U S A 96:8241-8246.

Dupret D, Revest JM, Koehl M, Ichas F, De Giorgi F, Costet P, Abrous DN, Piazza PV (2008) Spatial relational memory requires hippocampal adult neurogenesis. PloS ONE 3:e1959.

Farioli-Vecchioli S, Saraulli D, Costanzi M, Pacioni S, Cinà I, Aceti M, Micheli L, Bacci A, Cestari V, Tirone F (2008) The timing of differentiation of adult hippocampal neurons is crucial for spatial memory. PloS Biol 6:e246.

Fusani L, Metzdorf R, Hutchison JB, Gahr M (2003) Aromatase inhibition affects testosterone-induced masculinization of song and the neural song system in female canaries. J Neurobiol 54:370-379.

Gahr M (1990) Delineation of a brain nucleus: comparisons of cytochemical, hodological and cytoarchitectural views of the song control nucleus HVc of the adult canary. J Comp Neurol 294:30-36.

Gahr M (2004) Hormone-dependent neural plasticity in the juvenile and adult song system: what makes a successful male? Ann N Y Acad Sci 1016:684-703.

Goldman SA, Nottebohm F (1983) Neuronal production, migration, and differentiation in a vocal control nucleus of the adult female canary brain. Proc Natl Acad Sci U S A 80:2390-2394.

Gurney ME, Konishi M (1980) Hormone-induced sexual differentiation of brain and behavior in zebra finches. Science 208:1380-1383.

Güttinger HR (1985) Consequences of domestication on the song structures in the canary. Behaviour 94:254-278.

Hahnloser RH, Kozhevnikov AA, Fee MS (2002) An ultra-sparse code underlies the generation of neural sequences in a songbird. Nature 419:65-70.

Haubensak W, Narz F, Heumann R, Lessmann V (1998) BDNF-GFP containing secretory granules are localized in the vicinity of synaptic junctions of cultured cortical neurons. J Cell Sci 111:1483-1493.

Heid P, Güttinger HR, Prove E (1985) The influence of castration and testosterone replacement of the song architecture of canaries. Z Tierpsychol 69:224-237.

Hennequin LF, Thomas AP, Johnstone C, Stokes ES, Plé PA, Lohmann JJ, Ogilvie DJ, Dukes M, Wedge SR, Curwen JO, Kendrew J, Lambert-van der Brempt C (1999) Design and structure-activity relationship of a new class of potent VEGF receptor tyrosine kinase inhibitors. J Med Chem 42:5369-5389.

Huang EJ, Reichardt LF (2001) Neurotrophins: roles in neuronal development and function. Annu Rev Neurosci 24:677-736.

Imayoshi I, Sakamoto M, Ohtsuka T, Takao K, Miyakawa T, Yamaguchi M, Mori K, Ikeda T, Itohara S, Kageyama R (2008) Roles of continuous 
neurogenesis in the structural and functional integrity of the adult forebrain. Nat Neurosci 11:1153-1161.

Jarvis ED, Nottebohm F (1997) Motor-driven gene expression. Proc Natl Acad Sci U S A 94:4097-4102.

Jin H, Clayton DF (1997) Localized changes in immediate-early gene regulation during sensory and motor learning in zebra finches. Neuron 19:1049-1059.

Jin K, Zhu Y, Sun Y, Mao XO, Xie L, Greenberg DA (2002) Vascular endothelial growth factor (VEGF) stimulates neurogenesis in vitro and in vivo. Proc Natl Acad Sci U S A 99:11946-11950.

Kirn JR, Fishman Y, Sasportas K, Alvarez-Buylla A, Nottebohm F (1999) The fate of new neurons in adult canary high vocal center during the first 30 days after their formation. J Comp Neurol 411:487-494.

Kroodsma DE (1976) Reproductive development in a female songbird: differential stimulation by quality of male song. Science 192:574-575.

Leitner S, Voigt C, Garcia-Segura LM, Van't Hof T, Gahr M (2001) Seasonal activation and inactivation of song motor memories in wild canaries is not reflected in neuroanatomical changes of forebrain song areas. Horm Behav 40:160-168.

Li XC, Jarvis ED, Alvarez-Borda B, Lim DA, Nottebohm FA (2000) Relationship between behavior, neurotrophin expression, and new neuron survival. Proc Natl Acad Sci U S A 97:8584-8589.

Louissaint A Jr, Rao S, Leventhal C, Goldman SA (2002) Coordinated interaction of neurogenesis and angiogenesis in the adult songbird brain. Neuron 34:945-960.

Meshi D, Drew MR, Saxe M, Ansorge MS, David D, Santarelli L, Malapani C, Moore H, Hen R (2006) Hippocampal neurogenesis is not required for behavioral effects of environmental enrichment. Nat Neurosci 9:729-731.

Miller MM, McEwen BS (2006) Establishing an agenda for translational research on PTSD. Ann N Y Acad Sci 1071:294-312.

Mullen RJ, Buck CR, Smith AM (1992) NeuN, a neuronal specific nuclear protein invertebrates. Development 116:201-211.

Nottebohm F (1980) Testosterone triggers growth of brain vocal control nuclei in adult female canaries. Brain Res 189:429-436.
Nottebohm F, Stokes TM, Leonard CM (1976) Central control of song in the canary, Serinus canaris. J Comp Neurol 165:457-486.

Nottebohm F, Nottebohm ME, Crane L (1986) Developmental and seasonal changes in canary song and their relation to changes in the anatomy of song-control nuclei. Behav Neural Biol 46:445-471.

Nottebohm F, Nottebohm ME, Crane LA, Wingfield JC (1987) Seasonal changes in gonadal hormone levels of adult male canaries and their relation to song. Behav Neural Biol 47:197-211.

Pang PT, Teng HK, Zaitsev E, Woo NT, Sakata K, Zhen S, Teng KK, Yung WH, Hempstead BL, Lu B (2004) Cleavage of proBDNF by tPA/plasmin is essential for long-term hippocampal plasticity. Science 306:487-491.

Rasika S, Alvarez-Buylla A, Nottebohm F (1999) BDNF mediates the effects of testosterone on the survival of new neurons in an adult brain. Neuron 22:53-62.

Saxe MD, Battaglia F, Wang JW, Malleret G, David DJ, Monckton JE, Garcia AD, Sofroniew MV, Kandel ER, Santarelli L, Hen R, Drew MR (2006) Ablation of hippocampal neurogenesis impairs contextual fear conditioning and synaptic plasticity in the dentate gyrus. Proc Natl Acad Sci U S A 103:17501-17506.

Saxe MD, Malleret G, Vronskaya S, Mendez I, Garcia AD, Sofroniew MV, Kandel ER, Hen R (2007) Paradoxical influence of hippocampal neurogenesis on working memory. Proc Natl Acad Sci U S A 104:4642-4646.

Scharfman HE, Maclusky NJ (2005) Similarities between actions of estrogen and BDNF in the hippocampus: coincidence or clue? Trends Neurosci 28:79-85.

Vallet E, Kreutzer M (1995) Female canaries are sexually responsive to special song phrases. Anim Behav 49:1603-1610.

Voigt C, Leitner S (2008) Seasonality in song behavior revisited: seasonal and annual variants and invariants in the song of domesticated canary (Serinus canaria). Horm Behav 54:373-378.

Yamada K, Nabeshima T (2003) Brain-derived neurotrophic factor/TrkB signaling in memory processes. J Pharmacol Sci 91:267-270.

Yu AC, Margoliash D (1996) Temporal hierarchical control of singing in birds. Science 273:1871-1875. 